\title{
Experimental and Numerical Study of Melting of Particle-Laden Materials in a Cylinder ${ }^{*}$
}

\author{
Dawei Sun \\ Saint-Gobain High-Performance Materials \\ Northboro R\&D Center \\ Northboro, MA 01532 USA \\ S. Ravi Annapragada and Suresh V. Garimella ${ }^{\dagger}$ \\ School of Mechanical Engineering \\ Purdue University \\ West Lafayette, IN 47907-2088 USA
}

\begin{abstract}
The melting of a particle-laden slurry in a cylinder is investigated. The slurry consists of neutrally buoyant ceramic hollow spheres suspended in a paraffin wax. Melt front propagation and heat transfer processes during phase change in this particle-laden material is studied. The numerical analysis employs a particle-diffusive model and the enthalpy method. Experiments are carried out to validate the numerical model. The experimental boundary conditions are adequately represented with a constant and uniform side-wall temperature, a constant and uniform lower-surface temperature, and an adiabatic top wall. Reasonable agreement is obtained between the experiments and numerical predictions. It is found that the flow and heat transfer characteristics of the melt are greatly altered due to the presence of the solid particles and that the particle-diffusive model is insufficient to describe the particle migration during melting.
\end{abstract}

\section{Keywords}

Solid-liquid phase change, melting, natural convection, slurry, phase change material

\footnotetext{
${ }^{*}$ Submitted for possible publication in International Journal of Heat and Mass Transfer, January 2009

† To whom correspondence should be addressed: sureshg@purdue.edu, 765-494-5621
} 


\section{Nomenclature}

\begin{tabular}{|c|c|c|c|}
\hline$a$ & diameter of the particle & $\theta$ & nondimensional temperature, \\
\hline$c_{p}$ & specific heat & & $\theta=\left(T-T_{m}\right) /\left(T_{H^{-}} T_{m}\right)$ \\
\hline$d$ & diameter of the cylinder & $\rho$ & density \\
\hline$f_{L}$ & liquid fraction & \multicolumn{2}{|c|}{ Subscripts } \\
\hline$\vec{g}$ & gravitational vector & $a$ & actual \\
\hline$h$ & heat transfer coefficient & $B$ & base \\
\hline$H$ & height & $H$ & side wall \\
\hline$k$ & thermal conductivity & $i$ & initial \\
\hline$L$ & latent heat & $\infty$ & ambient \\
\hline$n$ & number of agglomerates & $L$ & liquid \\
\hline$p$ & pressure & $m$ & melting, measured \\
\hline$q^{\prime \prime}$ & heat flux & $\max$ & maximum \\
\hline \multirow[t]{2}{*}{$r$} & radius, coordinate in the radial & $N$ & Newtonian \\
\hline & direction & $p$ & particle \\
\hline$R$ & radius of the inner cylinder & ref & reference \\
\hline \multirow[t]{2}{*}{$R a$} & Rayleigh number, & $S$ & solid \\
\hline & $R a=g \beta\left(T_{H^{-}} T_{m}\right) R^{3} /(\alpha v)$ & $U$ & upper \\
\hline$t$ & time & & \\
\hline$T$ & temperature & & \\
\hline$u$ & velocity vector & & \\
\hline$z$ & coordinate in the vertical direction & & \\
\hline \multicolumn{4}{|c|}{ Greek } \\
\hline$\alpha$ & thermal diffusivity & & \\
\hline$\beta$ & thermal expansion coefficient & & \\
\hline$\delta$ & thickness of the polycarbonate wall & & \\
\hline$\varepsilon$ & artificial mushy zone thickness & & \\
\hline$\phi$ & particle volume fraction & & \\
\hline$\eta$ & dynamic viscosity & & \\
\hline$v$ & kinematic viscosity & & \\
\hline
\end{tabular}




\section{Introduction}

Particle-laden flows involving solid/liquid phase change are widely encountered in various scientific and engineering applications, such as manufacturing of novel energetic materials [1, 2], semi-solid castings [3-5], advanced energy storage [6, 7], and cryopreservation of biological cell suspensions [8], among others. While a number of theoretical and experimental investigations [7, $9,10]$ as well as numerical studies [3, 4, 11-15] have considered this problem, a satisfactory understanding of phase change involving particle-laden fluids has not been achieved to date.

The rheological behavior of concentrated suspensions under isothermal conditions has received considerable attention. A particle-diffusive model was described by Phillips et al. [9], in which a constitutive equation was derived for the particle flux. This model was later incorporated into a finite-volume numerical model by Fang and Phan-Thien [13] to study the behavior of concentrated suspensions in an eccentric circular geometry. A suspension-balance model was proposed by Nott and Brady [16], which takes the intensity of velocity fluctuations into account by linking the suspension pressure to the particle temperature. Implementation of such a model in a finite-volume framework was explored by Fang et al. [17]. Both the particlediffusive and the suspension-balance models are developed for isothermal fluids and do not adequately address the additional complexities introduced by the non-uniform thermal field and solid/liquid phase change.

While phase change processes in pure substances have been extensively studied (e.g., [18]), limited attention has been paid to the solidification/melting of slurries due to the complexity of the physical mechanisms involved, the incompleteness of the theoretical understanding, and limitations of the available numerical models. Semi-solid slurries, where particles and melt of the same substance coexist, are of particular practical interest. Through theoretical analysis, Fan et al. [19] studied the rheological behavior of semi-solid metals. The effects of electromagnetic stirring on phase change processes in semi-solid slurries was investigated by Chowdhury et al. $[20]$.

A slurry loaded with particles of an insoluble second phase behaves differently from semisolid slurries due to the presence of the insoluble solids. The Rayleigh-Benard stability of slurry solidification was investigated theoretically by Mackie [21], in which separate governing equations for the particles and the melt were formulated. Particle-particle interactions, however, 
were not taken into account. Hadji [22] analyzed the presence of a single foreign particle on the solid/liquid interface shape through an asymptotic approach. The study focused on the localized phenomena around a single particle, and the overall heat transfer characteristics of slurries were not analyzed. Using theoretical analysis, the effects of particle distribution on dendritic growth were studied in [23]. The dynamic changes in particle distribution and their effects on thermal fields were not explored. Almost all of these studies have addressed theoretical and numerical aspects of phase change processes in particle-laden flows. To the authors' knowledge, the heat transfer characteristics during the solidification or melting of particle-laden fluids have not been systematically explored through experimentally validated models. Experimental investigations can not only provide insight into the physical mechanisms involved, but also serve as benchmarks for the limited numerical models that have been developed for slurry transport.

The objective of the present work is to understand the phase-change heat transfer mechanisms characterizing the melting of particle-laden solids through experimental investigation and numerical modeling. A detailed experimental database has also been made available online ${ }^{\S}$, to facilitate future numerical model development and validation.

\section{Experimental Setup and Procedures}

The slurry considered in this work consists of silica-alumina ceramic microspheres (z-3125, $3 \mathrm{M})$ in a matrix of the paraffin wax, $n$-eicosane. The low melting point of $36.4^{\circ} \mathrm{C}$ for $n$-eicosane facilitates conduct of the experiment. The ceramic microspheres were chosen to match the low density of the matrix $\left(769 \mathrm{~kg} / \mathrm{m}^{3}\right)$ in the liquid phase to the extent possible so that the particles remain suspended in the melt for a longer time. It is noted that although the average density of the particles $\left(750 \mathrm{~kg} / \mathrm{m}^{3}\right)$ approximately matches that of the melt, there exists a distribution of densities in the particles. The thermophysical properties of the melt and the microspheres are given in Table 1. Properties of the particles were measured by Rob Hunter of 3M.

\subsection{Experimental Setup}

The experimental setup of Jones et al. [18] is used in the current work. A schematic diagram of the experimental setup is shown in Figure 1a. The slurry is filled into a cylindrical enclosure made of a polycarbonate cylindrical shell, an acrylic base and an acrylic block on top. The sides of the cylindrical enclosure are exposed to a hot water bath which is maintained at a

\footnotetext{
${ }^{\S}$ https://thermalhub.org/resources/188
} 
constant temperature by an immersion-type circulation heater. The dimensions of the enclosure are shown in Figure 1b. The bottom surface of the enclosure is maintained at a controlled temperature by means of a copper block pressed against the acrylic base with a thin layer of thermally conductive epoxy, which is in turn attached to a fan-cooled heat sink. A T-type thermocouple embedded in the cartridge heater provides feedback to a PID temperature controller which helps maintain a constant temperature at the bottom surface. The desired temperature is thus maintained by alternatively heating or cooling the copper block, based on the feedback from the controller. It is desired that the top surface of the slurry be well insulated so that the melting process is driven by bottom and radial heating. Although the effective thermal conductivity of the slurry $(k \approx 0.2 \mathrm{~W} / \mathrm{m}-\mathrm{K})$ and that of the acrylic top $(k=0.193 \mathrm{~W} / \mathrm{m}-\mathrm{K})$ are comparable, the heat conducted through the top acrylic block is shown to be less than $5 \%$ of the

total energy input to the phase change slurry based on readings from the thermocouples mounted in the acrylic block. Hence, an adiabatic boundary condition may be assumed at the top of the slurry domain. A narrow annular gap between the cylindrical shell and the top block as shown in Figure 1a allows the siphoning out of excess slurry resulting from expansion of the $n$-eicosane in the slurry as it melts.

Three thermocouple rakes (1, 2 and 3 shown in Figure 1b), each having 6 T-type thermocouples, are inserted into the domain of interest. Thermocouples mounted on the outer surface of the polycarbonate shell measure the temperature of the water bath. The uncertainty of the temperature measurements from these T-type thermocouples is $\pm 1.4^{\circ} \mathrm{C}$ [18]. Further details of the experimental setup are available in [18].

\subsection{Slurry Filling Procedure}

The main challenge while loading the enclosure with slurry is to maintain uniform slurry concentration in the entire domain. The problem is aggravated by segregation of lighter and heavier particles towards the top and bottom of the cylinder, respectively. A uniform slurry mixture, maintained at a uniform temperature of $60^{\circ} \mathrm{C}$ by placing it in a constant temperature oven, was found to completely segregate within $3000 \mathrm{~s}$ for a $5 \%$ slurry concentration. Longer segregation times were observed for higher concentrations (9000 s for $50 \%$ slurry) due to restriction of the motion of particles by neighboring particles. Filling the entire domain in one step therefore does not yield acceptable results. 
An alternative, batch-mode filling procedure, referred to as the "Beaker Method," is used. This is a standard laboratory method for preparing emulsions and is useful for low to moderate particle concentrations of slurries. Small quantities of the slurry are mixed in batches in a beaker. The mixed slurry is injected into the cylindrical enclosure and allowed to solidify in layers that are approximately $4 \mathrm{~mm}$ thick. Each layer is allowed a solidification time of 40-60 min before the next layer is added. Even though some segregation may take place over each 4 mm-thick layer and induce some local non-uniformity in the particle concentration, this effect is minimized due to the small thickness of each layer.

However, this method cannot be applied for the $50 \%$ particle concentration slurry due to jamming of the syringe. For this particle concentration, a different method was employed. The molten, uniformly mixed slurry is filled into the open cylindrical enclosure with the top block and the attached thermocouple rakes temporarily removed and replaced after the filling. The slurry is then frozen by surrounding the cylindrical enclosure with ice and water inducing high solidification rates and hence minimizing the time for particle segregation. Based on the thermocouple readings, the poured slurry solidifies within $500 \mathrm{~s}$. This method is effective for higher particle concentrations due to the lower segregation rates. A uniform particle distribution was observed through the entire cylinder when different cross sections of the solidified slurry were observed under a microscope.

\subsection{Experimental Procedure}

After the enclosure was loaded with slurry using one of the two methods discussed above, the temperature of the slurry was raised to a uniform higher temperature just below the transition temperature of $n$-eicosane $\left(\mathrm{T}_{\mathrm{t}}=36.2^{\circ} \mathrm{C}\right)$. In view of the uncertainty of the thermocouple readings, an initial temperature of $34.5^{\circ} \mathrm{C}$ was chosen for the solidified slurry. The desired initial temperature (higher than the prevalent ambient temperature of $20 \pm 3^{\circ} \mathrm{C}$ ) was achieved by filling the tank with water and supplying power to the cartridge heater. The heat sink fans were also turned on, as was the immersion-type circulation heater. The temperatures in the solidified slurry were allowed to reach steady-state values.

To start the melting process, the water in the enclosure was drained and quickly replaced with hot water. The immersion-type circulation heater served to maintain the temperature of the water bath at $70^{\circ} \mathrm{C}$. The bottom heater temperature was held at the initial temperature setting of $34.5^{\circ} \mathrm{C}$. Temperature readings were recorded at $15 \mathrm{~s}$ intervals. Four sets of experiments were 
performed, with particle concentrations of $0 \%, 5 \%, 25 \%$ and $50 \%$ by weight. The boundary conditions and initial temperatures were maintained the same in all the experiments. The $0 \%$ and $5 \%$ slurry experiments were performed twice to check for repeatability of the results. The time required for thermocouples to each reach an arbitrarily chosen temperature level of $55^{\circ} \mathrm{C}$ differed by less than $1.5 \%$ in each set of repeated experiments, showing good repeatability of the experimental procedures and measurements.

\section{Numerical Model}

The governing equations are derived based on a continuum approach, in which the details of micro- and macro-scale migration of the particles are described through an additional transport equation, following the procedure of Philips et al. [9]. With the following notations

$$
\langle\rho\rangle=\sum \rho_{i} \text {, and }\left\langle\rho u_{j}\right\rangle=\varphi_{i} \rho_{i} u_{i, j},
$$

the mass conservation equation takes a similar form as for a single phase:

$$
\frac{\partial}{\partial x_{j}}\left\langle\rho u_{j}\right\rangle=0
$$

$\varphi_{i}$ in Eq. (1) is the volume fraction of phase $i$. The momentum transport of the slurry follows the conventional Navier-Stokes equations:

$$
\frac{\partial\left\langle\rho u_{i}\right\rangle}{\partial t}+\frac{\partial}{\partial x_{j}}\left\langle\rho u_{i} u_{j}\right\rangle=-\frac{\partial}{\partial x_{i}} p+\frac{\partial}{\partial x_{j}} S_{i j}
$$

where $S$ is the fluid stress tensor. The effect of the presence of the particles on bulk fluid momentum transport manifests itself through a change in the rheology of the fluid. In this work, the Krieger-Dougherty formulation is used to relate the particle concentration to the effective viscosity [24]:

$$
\eta=\eta_{N}\left(1-\frac{\phi}{\phi_{m}}\right)^{\alpha}
$$

where $\eta_{N}$ is the viscosity of the melt, and $\alpha=-1.82$. The maximum packing ratio $\phi_{\max }=0.60$ of mono-size spheres was found through experiments.

The heat transfer characteristics of the slurry are also strongly affected by the presence of the solid particles. The effective properties, such as thermal conductivity and specific heat of the mixture, are altered. In this study, the melt ( $n$-eicosane) and the particulate phase (micro- 
balloons) are carefully chosen to have similar thermophysical properties (Table 1). Thus the slurry samples in this work are assumed to be in thermal equilibrium. Under this assumption, the continuum mass, momentum and energy conservation equations are applicable for the slurry. Hence the energy equation can be written as:

$$
\left\langle\rho c_{P}\right\rangle\left(\frac{\partial T}{\partial t}+\nabla \cdot(\langle u\rangle T)\right)=\nabla \cdot(\langle k\rangle \nabla T)+S_{T}+L \frac{\partial\left(f_{l} \phi\right)}{\partial t}
$$

where $S_{T}$ is the source term introduced by the enthalpy-porosity model and the term $L \partial\left(f_{l} \phi\right) / \partial t$ is introduced to account for the presence of the solid particles.

The shear-induced particle migration is driven by the concentration gradients and by the shear rate gradients. The phenomenological model developed by Philips et al. [9] was adopted in this work, which leads to the following equation for particle migration

$$
\frac{\partial \phi}{\partial t}+u_{j} \frac{\partial \phi}{\partial x_{j}}=\nabla \cdot\left(\Gamma_{\phi} \frac{\partial \phi}{\partial x_{j}}\right)+S_{\phi}
$$

The diffusive coefficient $\Gamma_{\phi}$ and source term $S_{\phi}$ can be written as:

$$
\Gamma_{\phi}=a^{2} \phi\left(K_{\mathrm{c}}+K_{\eta} \phi \frac{\partial \eta}{\eta \partial \phi}\right) \dot{\gamma}, \text { and } S_{\phi}=K_{c} a^{2} \phi^{2} \nabla \dot{\gamma}
$$

where $a$ denotes the particle diameter, $\dot{\gamma}$ the shear rate and coefficients $K_{c}=0.41$ and $K_{\eta}=0.62$, respectively.

The coupled equations (2), (3), (5) and (6) are solved using the commercial software package, FLUENT, with the particle transport equation (6) introduced using the user-defined scalar (UDS) functionality and the last term in Eq. (5) treated as a source term.

No-slip boundary conditions for the velocity are assumed on all solid boundaries. The normal particle flux at the wall is zero.

\section{Results and Discussion}

The melting behavior of particle-laden solids in a cylinder is discussed. The effects of solid particle concentration are explored by comparing the transient temperature distributions and velocity fields at four different particle concentrations, i.e., $\phi=0 \%, 5 \%, 25 \%$ and $50 \%$. Experimental measurements are presented along with numerical predictions.

\subsection{Experimental Observations}


The melting of a pure substance under the present set of experimental conditions goes through conduction-dominant, convection-dominant and shrinking-solid stages as detailed in [18]. Similar stages are observed in the present slurry experiments as well. Figure 3 through Figure 5 show the transient temperature histories for the melting of various concentrations of slurries at selected locations along the outer, middle and center thermocouple rakes, respectively. Distinct differences can be seen among cases with the different particle loadings. This can be attributed to the particle agglomeration effects. As in the case of the casting of semi-solid metal slurries, dynamic interactions between solid particles in the slurry under consideration result in the formation of agglomerates. The paraffin can become entrapped in the agglomerates during the slurry preparation and the melting process, depending on the solid fraction. As a consequence, the effective fraction of the solid suspensions increases due to the solid-like behavior of the entrapped liquid under shear flow. As explained in [25], the effective solid volume fraction, defined as $\phi_{\mathrm{eff}}=\phi+\phi_{\mathrm{liq}}=[1+(n-1) / n A] \phi$, is affected by the solid volume fraction $(\phi)$, the agglomerate size $(n)$ and the packing mode $(A)$ in the agglomerates. The parameters $n$ and $A$ are generally determined experimentally [19, 25].

For dilute slurries $(\phi \leq 5 \%)$, clustering effects can be neglected due to the weaker interactions between the solid particles. The length scales for both the phase change process and natural convection are then determined only by the size of the container, i.e., the diameter of the cylinder. Hence, the strength of buoyancy-induced natural convection, as in the case of pure n-eicosane ( $\phi$ $=0 \%$ ), can be characterized based by $R a=\rho g \beta \Delta T d^{3} / \eta_{N} \alpha$. The effect of suspension concentration on slurry solidification manifests only through changes in the effective properties. As shown in Figure 2, the apparent viscosity of the slurry with $\phi=5 \%$ is only $17 \%$ greater than for the pure material with $\phi=0 \%$. This explains the similarities in melting behavior of the dilute slurry and pure wax revealed by the temperature histories from the thermocouple rakes. The presence of the solid particles reduces the apparent latent heat of the slurry, resulting in an increase in the Stefan number as $\phi$ increases. Assuming SteFo to be an approximate time scale for the melting process, $\phi=5 \%$ will lead to a slower melting rate compared to the case of pure wax. Consequently, the natural convection-dominant stage is slightly suppressed. Detailed datasets for the comparison are provided as supplemental information for ease of use in benchmarking numerical models. 
From Figure 3 through Figure 5, it can be seen that the melting behavior of the more highly loaded slurries ( $\phi=25 \%$ and $50 \%$ ) is significantly different from that of the pure melt and the dilute slurry. For the denser slurries $(\phi \geq 25 \%)$, the entrapped liquid in agglomerates can no longer be ignored, i.e., $\mathrm{O}\left(\phi_{\text {liq }}\right) \sim \mathrm{O}\left(\phi_{\text {eff }}\right)$. Thus the errors in the predicted results based on the assumption of $\phi_{\mathrm{eff}} \approx \phi$ can be significant. Agglomerates may form and break up under the viscous shear forces. As the particle fraction is increased, the melt convection is less affected by the size of the container and is increasingly determined by interactions within an individual agglomerate or between neighboring agglomerates. As a result, the apparent viscosity of the slurry increases exponentially as $\phi$ increases (Figure 2). In these denser slurries, the higher solid particle fraction (and the commensurate higher effective viscosity) leads to a decrease in the strength of convection in the slurry. In such a case, a particle Rayleigh number, $R a_{p}=\rho g \beta \Delta T a^{3} / \eta_{N} \alpha$, can be used to estimate the effects of natural convection $\left(R a_{p, e f f, 25 \%}=\right.$ $2.23 \times 10^{-1}$ and $R a_{p, e f f, 50 \%}=1.88 \times 10^{-2}$ ). The higher the $\phi$, the larger is the $\eta_{N}$ and consequently the smaller is $R a_{p}$. When the particle loading approaches the packing limit ( $\phi=68 \%$ ), a dramatic increase in effective viscosity is expected as there can be no movement of the solid particles and the path for flow of pure n-eicosane through the solid particles becomes very tortuous. Thus the overall phase change heat transfer process is dominated by heat conduction, which is in sharp contrast with the case of the pure melt.

At the particle loading of $\phi=25 \%$, the competition between conduction and melt convection causes the overall heat transfer characteristics to fall between the dilute $(\phi=5 \%)$ and dense $(\phi=$ $50 \%$ ) slurry cases. A stronger influence of convection is observed at $\phi=25 \%$ than that at $\phi=$ $50 \%$. The non-uniform particle distribution caused by shear-induced particle migration leads to convection-dominant heat transfer in certain regions (low $\phi$ ) and conduction-dominant in others (high $\phi$ ) within this slurry. This explains the transient temperature histories at B1, D1 and F1 shown in Figure 5.

For the most dense slurry $(\phi=50 \%)$, temperatures at each TC rake reach the melting point roughly at the same time (Figure 3 to Figure 5), indicating a flat solid/liquid interface. This is a clear feature of a conduction-dominated process.

The transient heat transfer behavior of the slurry during melting can be further analyzed by examining the temperature variations along the TC rakes at different times. Since it is difficult 
to correlate melting time against nondimensional parameters such as Stefan number or Fourier number [18], and the complete melting process cannot be visually observed due to the high particulate content, it is reasonable to use the time taken, $t_{0}$, for the last TC (A1) in the slurry to reach melting point as the characteristic time scale. The time scales for different cases are listed in Table 2. It takes longer for the denser slurries to melt completely relative to the dilute cases. This can be attributed to the reduced natural convection as the particle concentration increases even though the effective mass of wax to be melted is reduced. The temperature profiles along different TC rakes at $t=0.25 t_{0}, 0.50 t_{0}$ and $0.75 t_{0}$, illustrate this point in Figure 6 . The $0 \%$ and $5 \%$ particle loading cases (Figure 6(a)-(b)) behave very similarly, with strong convection effects being evident. At $t=0.25 t_{0}$, the center and middle rakes are still dominated by heat conduction while the effects of convection are significant at the outer rake. From time $t=0.50 t_{0}$ onwards, the entire domain is in a convection-dominant regime.

For cases with higher particle loading ( $\phi \geq 25 \%)$, the effects of melt convection manifest much later in the process than for the dilute case $(\phi \leq 5 \%)$. The temperature profiles in these cases are more conduction-dominated (Figure 6c). The predominant heat transfer mechanism seen by the center rake during the entire melting process in these cases is conduction. For the middle rake, convection effects are observed only at $t=0.75 t_{0}$. As discussed above, heat conduction is the dominant heat transfer mode as the particle loading approaches packing limits. This is clearly reflected in Figure 6d, in which a linear inward propagation of isotherms from the outer rakes to the center rake is observed.

\subsection{Numerical Simulation}

\subsubsection{Model validation}

In order to validate the numerical analysis, the model is first employed in the circular Couette flow calculation illustrated in Figure 7a, for which detailed experimental data are available [9]. The suspension considered consists of polymethyl methacrylate (PMMA) spheres suspended in a Newtonian oil (consisting of $14.07 \%$ wt practical grade 1,1,2,2 tetrabromoethane, $35.66 \% \mathrm{wt}$ UCON oil (H-90 000), and 50.27\% Triton X-100, an alkylaryl polyether alcohol). The radii of the inner and outer cylinder are given by $r$ and $R$, respectively. In this exercise, $R=4 r$ is used. The inner cylinder rotates with an angular velocity $\omega$, while the outer cylinder is stationary.

At steady state, the flow is unidirectional, and the velocity and concentration profile along the radial direction are given by the following analytical formulation [9] 


$$
16 r^{2}=\frac{\phi}{\phi_{m}}\left(\frac{1-\phi_{w} / \phi_{m}}{1-\phi / \phi_{m}}\right)^{\varepsilon\left(K_{\eta} / K_{c}-1\right)}
$$

and

$$
\frac{d}{d r}\left(\frac{u_{\theta}}{r}\right)=\frac{C}{r^{2}}\left(1-\frac{\phi}{\phi_{m}}\right)^{\varepsilon}
$$

Using the numerical model developed in the present work, the velocity as well as particle concentration fields are calculated and compared with analytical solutions as shown in Figure $7 \mathrm{~b}$. The comparisons show excellent agreement between the numerical predictions and the analytical solution.

\subsubsection{Modeling melting of slurry}

The numerical model developed in Section 3 is employed to study the melting process of the slurry. This model has previously been extensively validated against experiments for the case of melting of pure $n$-eicosane [18].

A comparison of predicted temperatures with measurements at selected locations for cases with dilute particle loading $(\phi=5 \%)$ shows reasonable agreement in Figure 8 . Any shearinduced particle migration occurring in this dilute slurry is thus captured reasonably well in the model. This is in part due to the fact that the $5 \%$ slurry has quite similar viscosity to the pure melt for which the model has been demonstrated to be effective. The heat transfer characteristics of these two cases also show great similarity. A detailed discussion of the pure substance behavior is available in [18].

As the particle concentration is increased, the effects of particle migration become significant. For the particle loading of $\phi=25 \%$, the numerical predictions deviate from the thermocouple readings with much poorer agreement than for the dilute case (Figure 9). For measurements along the outer rake, where conduction is still dominant and the effects of shear-induced particle migration may be ignored, the agreement is relatively better. The mismatch becomes significant with TCs along the center rake, which is the region that experiences phase change much later and hence suffers from greater particle migration effects. This implies that the particle diffusive model adopted in this work does not accurately capture the particle distribution changes. 
For the most highly loaded slurry considered ( $\phi=50 \%$, Figure 10), the model again provides better agreement with the measurements. As discussed previously, the slurry is very viscous in this case and hence heat conduction is the dominant phase change heat transfer mechanism. Thus the particle migration due to shear flow is not as significant as in the other two cases, and a conduction model is adequate.

The discrepancy between the experimental measurements and the numerical predictions from the model developed here can be attributed to several factors. The assumptions made in the model are a major factor: The model is based on the assumption of neutrally buoyant particles, which is not the case in the experiment due to the slight difference in the fluid and particle densities $\left(\delta=\left(\rho_{s}-\rho_{p}\right) / \rho_{s}=5 \%\right.$ as seen from Table 1$)$. The particle migration due to buoyancy also contributes to some of the discrepancies observed. The large difference in specific heat values of the wax and the microspheres is another important contributor. While effective values are used for other properties to reflect the dynamic changes in particle content, a constant, averaged value is used for specific heat due to the limitations of the model. The differences in specific heat between the wax and particles may also lead to non-equilibrium phase change, which is ignored in the current model.

As discussed in Section 4.1, the effective particle volume fraction is given by $\phi_{\mathrm{eff}}=\phi+\phi_{\mathrm{liq}}=$ $[1+(n-1) / n A] \phi$. Due to the lack of information on the actual rheological behavior of the suspensions, the assumption $\phi_{\mathrm{eff}} \approx \phi$ is adopted in the numerical model. This is a reasonable assumption for dilute slurry $(\phi<10 \%)$ due to the relatively weak clustering effects. For denser suspensions ( $\phi=25 \%$ and $50 \%$ ), the entrapped liquid in agglomerates can no longer be ignored, i.e., $\mathrm{O}\left(\phi_{\text {liq }}\right) \sim \mathrm{O}\left(\phi_{\text {eff }}\right)$. Thus the errors in the predicted results introduced by $\phi_{\text {eff }} \approx \phi$ could be significant.

The degree of subcooling of the slurry is another contributor to the discrepancy between prediction and experiment. The initial solidified slurry is at a slightly subcooled temperature in the current experimental setup. The degree of subcooling $\theta_{u}=\left(\mathrm{T}_{\mathrm{i}}-\mathrm{T}_{\mathrm{m}}\right) /\left(\mathrm{T}_{\mathrm{H}-} \mathrm{T}_{\mathrm{m}}\right)$ is very small and hence slight variations in $\theta$ can result in significant changes in the starting time of the melting process, and consequently in the temperature history at any given location. The small initial subcooling combined with heat losses from the surfaces leads to deviations from the numerically predicted temperature histories. 
The particle diffusive model adopted in this work appears to be inadequate in modeling the rheological behavior of the slurry, and improved models should be developed. Rigorous experimental measurements can help inform the development of more realistic models.

\section{Conclusions}

The solid/liquid phase change processes in particle-laden fluids are investigated by means of experiments and numerical modeling. The effects of different particle loading percentages on the temperature histories in the domain are explored.

The experimental measurements show that the slurry with lower particle loading ( $\phi=5 \%)$ behaves in a manner similar to melting of pure wax. Strong melt convection effects are observed in both these cases. For a heavily loaded slurry $(\phi=50 \%)$, the melting process is dominated by heat conduction. A transition from convection- to conduction-dominant effects is observed in the slurry with intermediate particle loading ( $\phi=25 \%)$.

It is also found that the diffusive flux model based on isothermal experiments is not adequate for describing the melting of slurries with different particle loadings. The model provides reasonable agreement for the dilute slurry $(\phi=5 \%)$ which displays only mild particle-loading effects as well as for the very dense slurry $(\phi=50 \%)$ which undergoes conduction-dominated. More advanced numerical models are needed to more accurately predict the phase change heat transfer behavior of particle-laden fluids.

\section{Acknowledgement}

Financial support for this work from the US Army Armaments Research Development Engineering Center (ARDEC) at Picatinny Arsenal is gratefully acknowledged. Rob Hunter of $3 \mathrm{M}$ characterized the particles used in the slurry solidification experiments.

\section{References}

[1] D. Sun, S. V. Garimella, N. Naik, S. K. Singh, Numerical and Experimental investigation of the melt casting of explosives, Propellants, Explosives, Pyrotechnics, 30 (2005) 369-380. 
[2] S. A. Annapragada, D. Sun, S. V. Garimella, Prediction of effective thermo-mechanical properties of particulate energetic materials, Computational Materials Science, 40 (2007) 255266.

[3] J. Chowdhury, S. Ganguly, S. Chakraborty, Numerical simulation of transport phenomena in electromagnetically stirred semi-solid materials processing, Journal of Physics, D: Applied Physics, 38 (2006) 2869-2880.

[4] L. Orgeas, J. P. Gabathuler, T. Imwinkelried, C. Paradies, M. Rappaz, Modelling of semisolid processing using a modified temperature-dependent power-law model, Modelling and Simulation in Materials Science and Engineering (2003) 553-574.

[5] Z. Fan, Semisolid metal processing, International Materials Review, 47 (2002) 49-85.

[6] H. Indaba, C. Dai, A. Horibe, Natural convection heat transfer of microemulsion phasechange-material slurry in rectangular cavities heated from below and cooled from above, Int J. Heat Mass Transfer, 46 (2003) 4427-4438.

[7] H. Indaba, M.-J. Kim, A. Horibe, Melting heat transfer characteristics of microencapsulated phase change material slurries with plural microcapsules having different diameters, Trans ASME J. Heat Transfer, 126 (2004) 558-565.

[8] C. Korber, G. Rau, M. D. Cosman, E. G. Cravalho, Interaction of particles and a moving iceliquid interface, Journal of Crystal Growth, 72 (1985) 649-662.

[9] R. J. Phillips, R. C. Armstrong, R. A. Brown, A. L. Graham, J. R. Abbott, A constitutive equation for concentrated suspension that account for shear induced particle migration, Phys. Fluids A, 4 (1992) 30-40.

[10] J. R. Abbott, N. Tetlow, A. L. Graham, S. A. Altobelli, E. Fukushima, L. A. Mondy, T. S. Stephens, Experimental observations of particle migration in concentrated suspensions: Couette flow, J. Rheol., 35 (1991) 773-795.

[11] J. F. Brady, G. Bossis, The rheology of concentrated suspensions of spheres in simple shear flow by numerical simulation, J. Fluid Mechanics, 155 (1985) 105-129.

[12] J. F. Brady, G. Bossis, Stokesian Dynamics, Ann. Rev. Fluid Mechanics, 20 (1988) 111-157.

[13] Z. Fang, N. Phan-Thien, Numerical simulation of particle migration in concentrated suspensions by a finite volume method, J. Non-Newtonian Fluid Mechanics, 58 (1995) 67-81.

[14] Z. Fang, N. Phan-Thien, A particle suspension model: an unstructured finite-volume implementation, J. Non-Newtonian Fluid Mechanics, 80 (1999) 135-153. 
[15] C. J. Ho, J. F. Lin, S. Y. Chiu, Heat transfer of solid-liquid phase-change material suspensions in circular pipes: effects of wall conduction, Numerical Heat Transfer, Part A, 45 (2004) 171-190.

[16] P. R. Nott, J. F. Brady, Pressure driven flow of suspensions: simulation and theory, J. Fluid Mechanics, 275 (1994) 157-199.

[17] Z. Fang, A. A. Mammoli, J. F. Brady, M. S. Ingber, L. A. Mondy, A. L. Graham, Flowaligned tensor model for suspension flow, Int J. Multiphase Flow, 28 (2002) 137-166.

[18] B. J. Jones, D. Sun, S. Krishnan, S. V. Garimella, Experimental and numerical study of melting in a cylinder, International Journal of Heat and Mass Transfer, 49 (2006) 2724-2738.

[19] Z. Fan, J. Y. Chen, Modelling of rheological behaviour of semisolid metal slurries - Part 2 Steady state behaviour, Materials Science and Technology, 18 (2002) 243-249.

[20] J. Chowdhury, S. Ganguly, S. Chakraborty, Numerical simulation of transport phenomena in electromagnetically stirred semi-solid materials processing, Journal of Physics, D: Applied Physics, 38 (2005) 2869-2880.

[21] C. Mackie, Convective stability of a particle-laden fluid system in the presence of solidification, Int J. Heat Mass Transfer, 43 (2000) 1617-1627.

[22] L. Hadji, Axisymmetric shapes and forces resulting from the interaction of a particle with a solidifying interface, Physical Review E, 66 (2002) 041404.

[23] B. Dutta, M. K. Surappa, Directional dendritic solidification of a composite slurry: Part II. Particle distribution, Metallurgical and Materials Transactions A: Physical Metallurgy and Materials Science, 29A (1998) 1329-1339.

[24] I. M. Krieger, Dimensional approach to colloid rheology, Transactions of the Society of Rheology 7(1963) 101-109.

[25] J. Y. Chen, Z. Fan, Modelling of rheological behaviour of semisolid metal slurries, Part 1 theory, Materials Science and Technology, 18 (2002) 237-242. 


\section{Figure Captions}

Figure 1. (a) Schematic diagram of the experimental facility, and (b) cylindrical enclosure dimensions and boundary conditions. Inset in (a) shows an expanded view of the top of the test cell and the locations of the thermocouples imbedded in the acrylic top. The thermocouples are approximately $0.5 \mathrm{~mm}$ and $3.8 \mathrm{~mm}$ from the lower surface of the acrylic top. An enlarged view of the test cylinder is shown in (b). The polycarbonate side wall is at a constant temperature $\mathrm{T}_{\mathrm{H}}$ on the outside, the acrylic bottom wall is at a constant temperature $T_{B}$ on the outside, and the top wall is adiabatic. The radial edge of the acrylic bottom wall is insulated as shown.

Figure 2. Predicted apparent viscosities [25] as a function of particle volume fraction. The values shown in the figure are at $\phi=5 \%, 25 \%$ and $50 \%$, respectively.

Figure 3. Comparison of temperature histories along the outer TC rakes B3, D3 and F3 (see Figure 1b) for cases with different particle loadings.

Figure 4. Comparison of temperature histories along the middle TC rakes, B2, D2, and F2 (see Figure 1b), for cases with different particle loadings.

Figure 5. Comparison of temperature histories along the center TC rakes, B1, D1 and F1 (see Figure 1b), for cases with different particle loadings.

Figure 6. Transient temperature profiles along different thermocouple rakes for cases with different particle loadings, (a) $\phi=0 \%$, (b) $\phi=5 \%$, (c) $\phi=25 \%$, and (d) $\phi=50 \%$.

Figure 7. (a) Schematic diagram of the circular Couette flow problem, and (b) comparison between analytical and predicted velocity and particle distribution profiles.

Figure 8. Comparison of predicted and measured temperature variations at selected TC locations for $\phi=5 \%$.

Figure 9. Comparison of predicted and measured temperature variations at selected TC locations for $\phi=25 \%$.

Figure 10. Comparison of predicted and measured temperature variations at selected TC locations for $\phi=50 \%$. 
Table 1. Thermophysical properties of the phase change material and the microspheres.

\begin{tabular}{rlcc}
\hline Property & Units & $n$-eicosane [18] & Particles \\
\hline Thermal conductivity, $k$ & $\mathrm{~W} / \mathrm{m}-\mathrm{K}$ & 0.146 & 0.25 \\
Specific heat, $c_{p}$ & $\mathrm{~J} / \mathrm{kg}-\mathrm{K}$ & 2400 & 240 \\
Density, $\rho$ & $\mathrm{kg} / \mathrm{m}^{3}$ & 839.5 & 800 \\
\hline
\end{tabular}


Table 2. Experimetal times used for the comparisons in Figure 6; $t_{0}$ refers to the characteristic time scale of the melting process.

\begin{tabular}{lcccc}
\hline$\phi$ & $0.25 \mathrm{t}_{0}(\mathrm{~s})$ & $0.50 \mathrm{t}_{0}(\mathrm{~s})$ & $0.75 \mathrm{t}_{0}(\mathrm{~s})$ & $\mathrm{t}_{0}(\mathrm{~s})$ \\
\hline $0 \%$ & & & & 3176 \\
$5 \%$ & 791 & 1583 & 2374 & 3166 \\
$25 \%$ & 1009 & 2018 & 3027 & 4036 \\
$50 \%$ & 1120 & 2240 & 3360 & 4480 \\
\hline
\end{tabular}




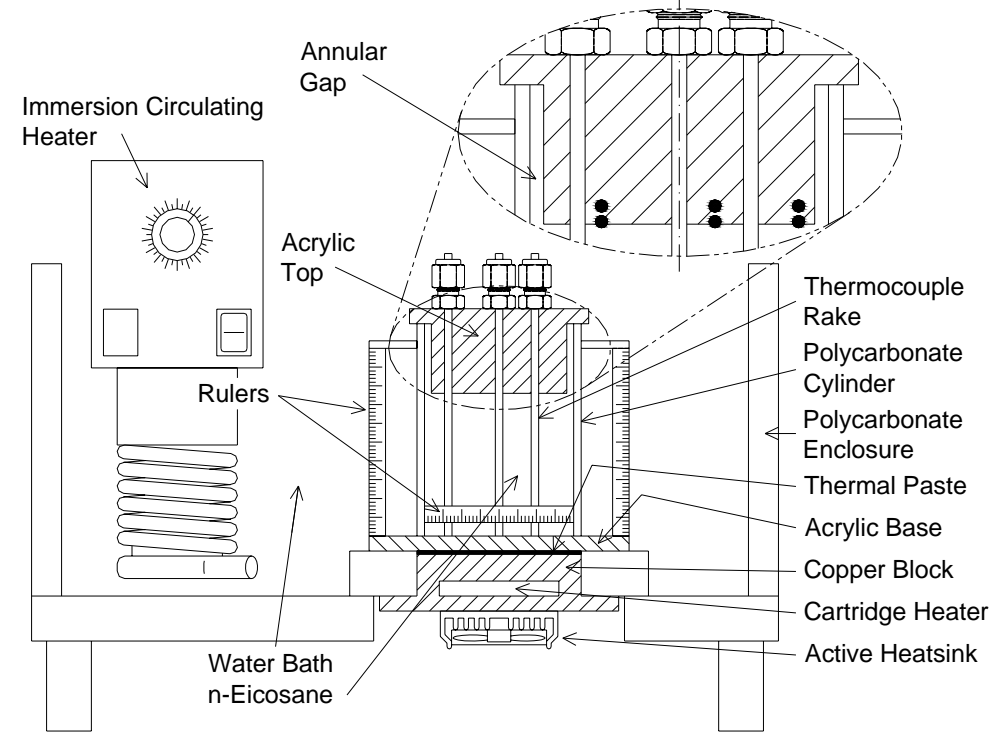

(a)

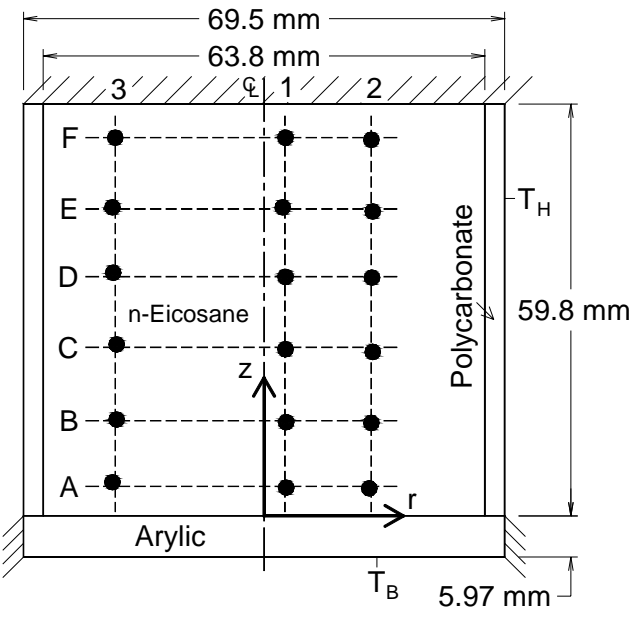

(b)

Figure 1. (a) Schematic diagram of the experimental facility, and (b) cylindrical enclosure dimensions and boundary conditions. Inset in (a) shows an expanded view of the top of the test cell and the locations of the thermocouples imbedded in the acrylic top. The thermocouples are approximately $0.5 \mathrm{~mm}$ and $3.8 \mathrm{~mm}$ from the lower surface of the acrylic top. An enlarged view of the test cylinder is shown in (b). The polycarbonate side wall is at a constant temperature $T_{H}$ on the outside, the acrylic bottom wall is at a constant temperature $T_{B}$ on the outside, and the top wall is adiabatic. The radial edge of the acrylic bottom wall is insulated as shown. 


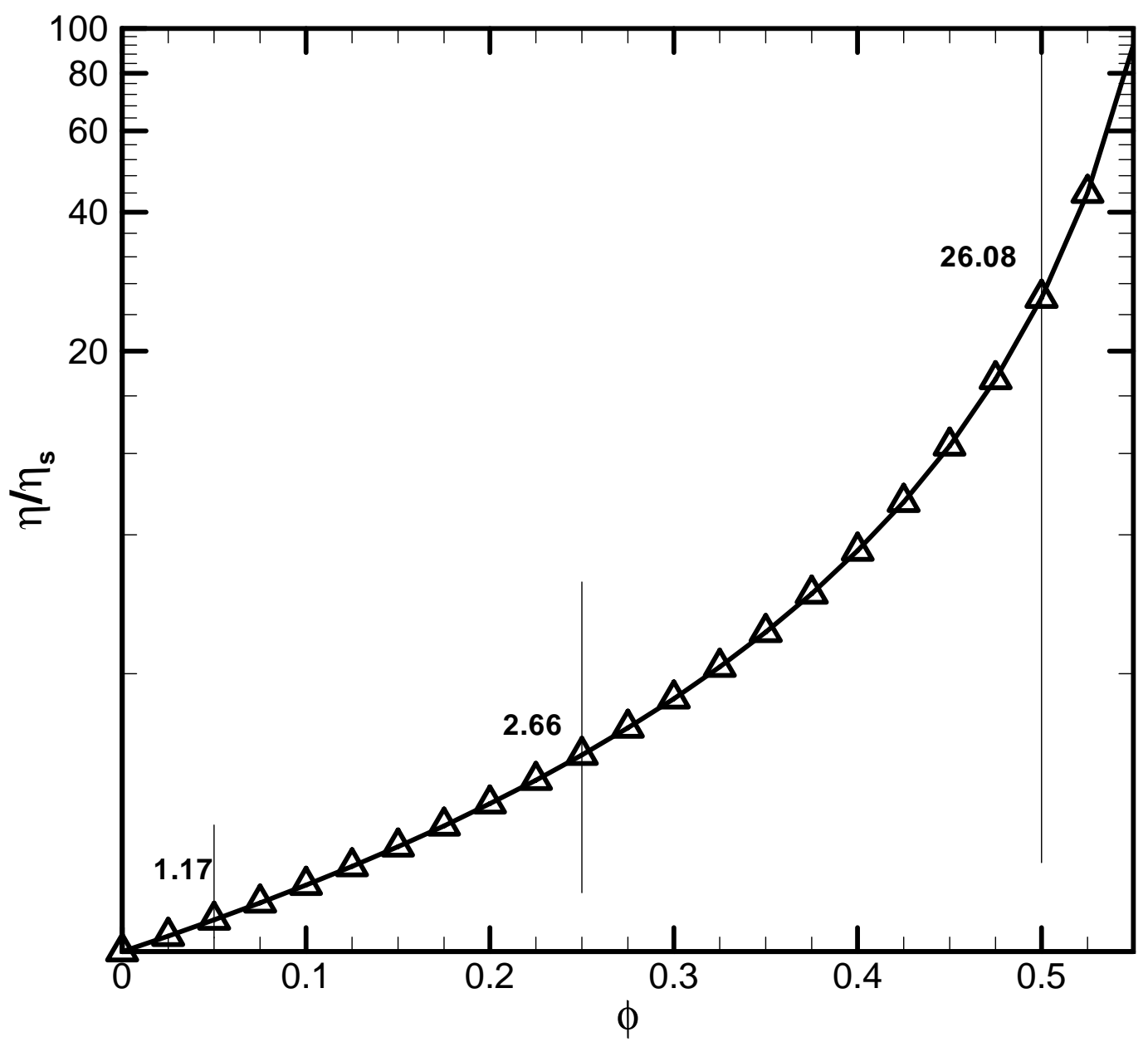

Figure 2. Predicted apparent viscosities [25] as a function of particle volume fraction. The values shown in the figure are at $\phi=5 \%, 25 \%$ and $50 \%$, respectively. 


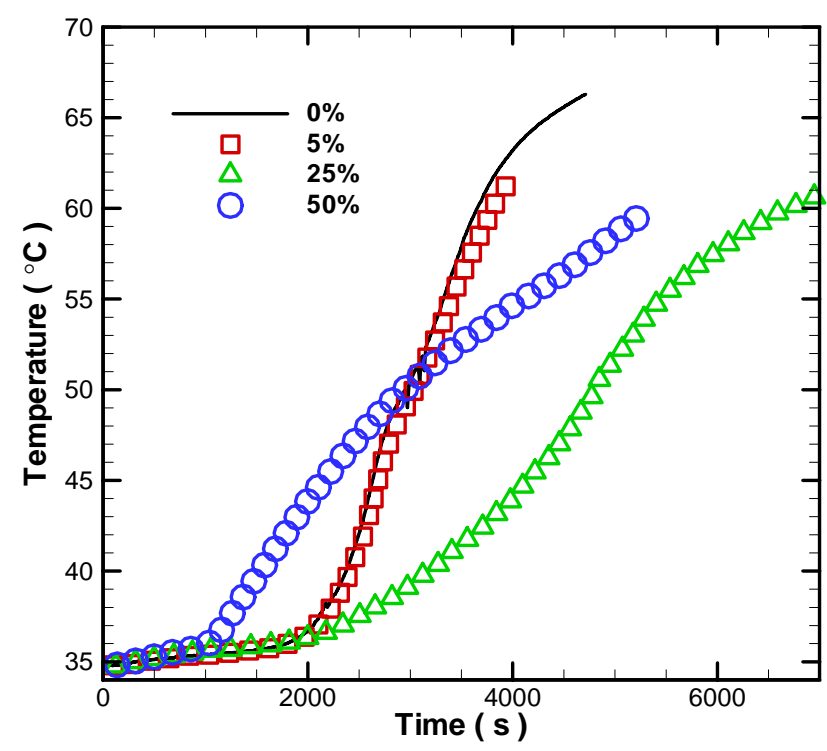

(a) B3

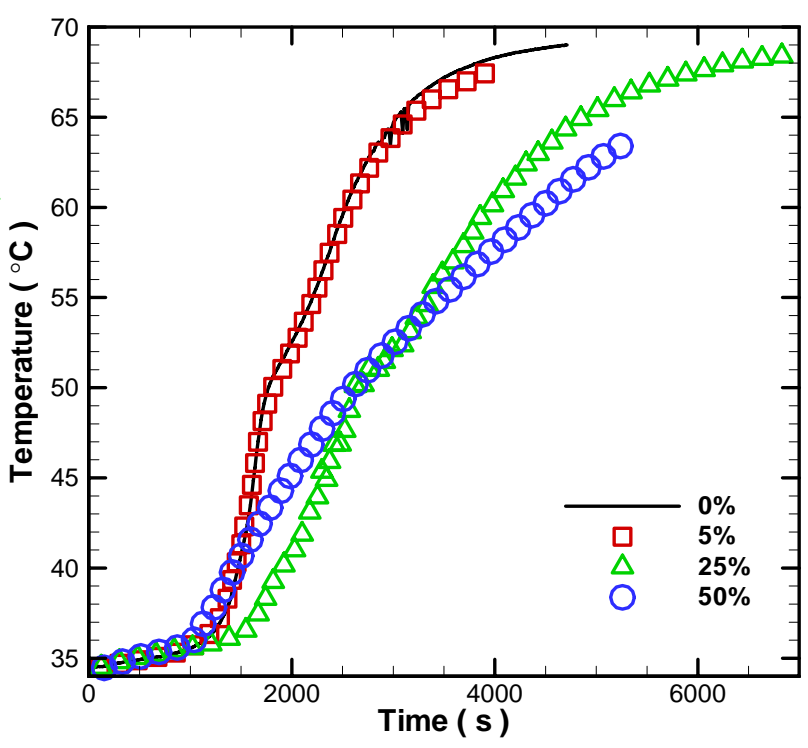

(b) D3

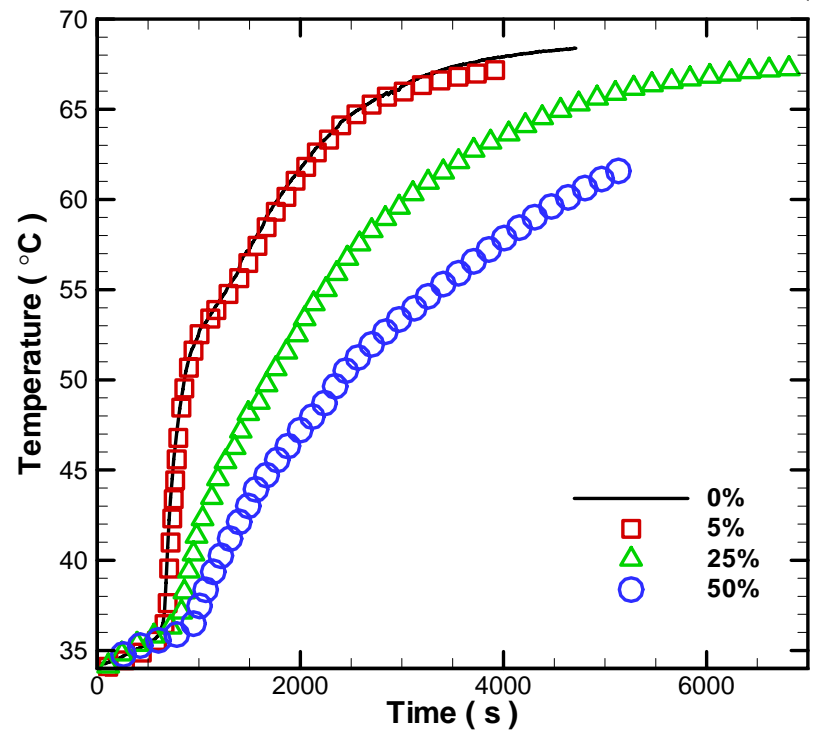

(c) F3

Figure 3. Comparison of temperature histories along the outer TC rakes B3, D3 and F3 (see Figure 1b) for cases with different particle loadings. 


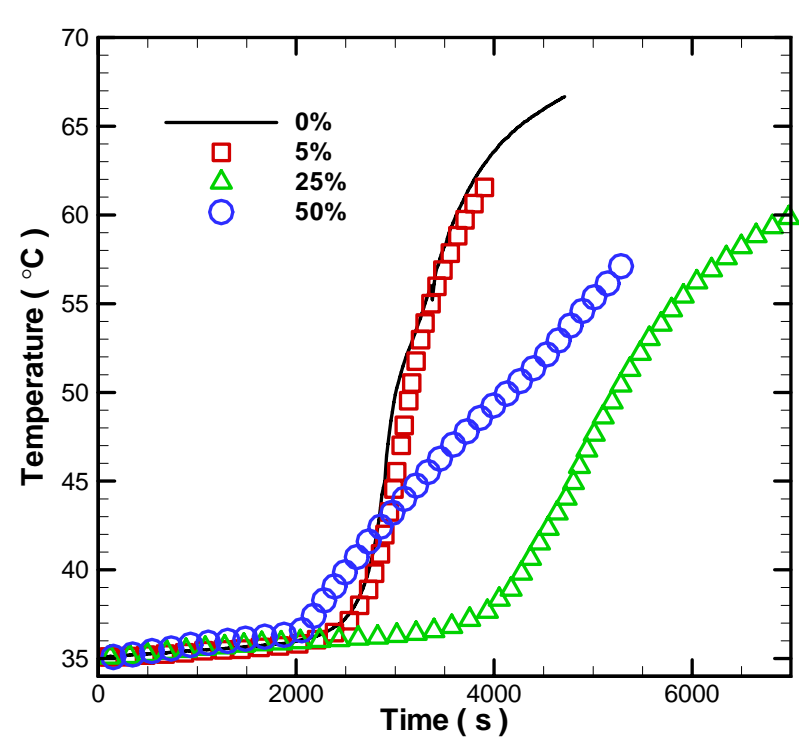

(a) B2

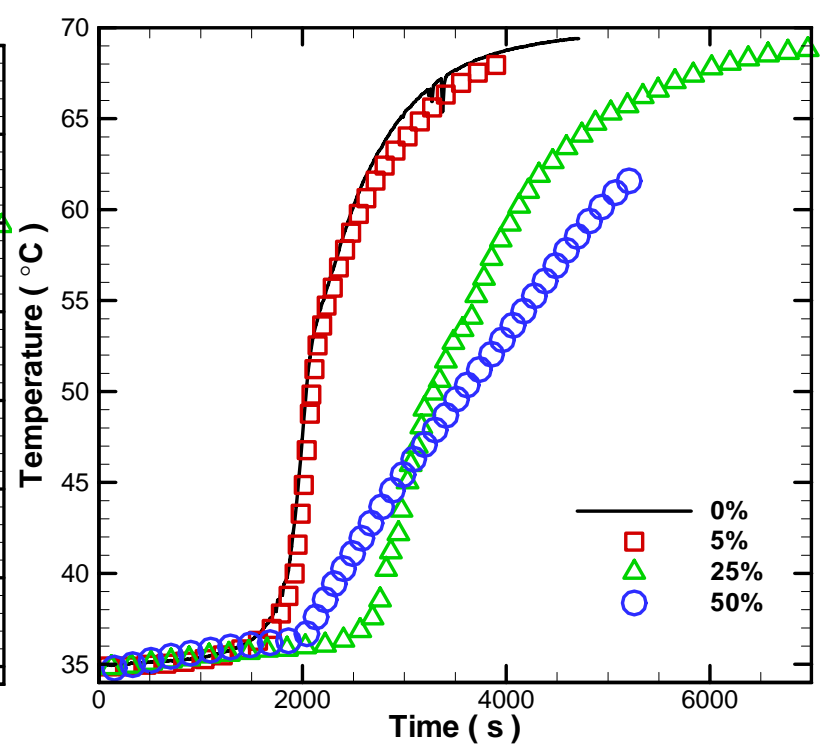

(b) D2

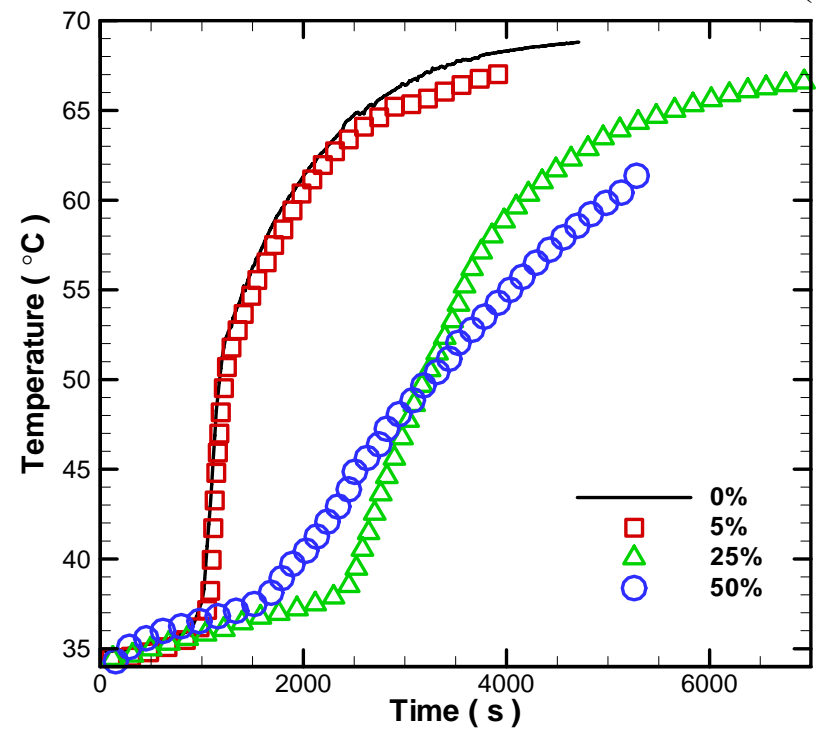

(c) F2

Figure 4. Comparison of temperature histories along the middle TC rakes, B2, D2, and F2 (see Figure 1b), for cases with different particle loadings. 


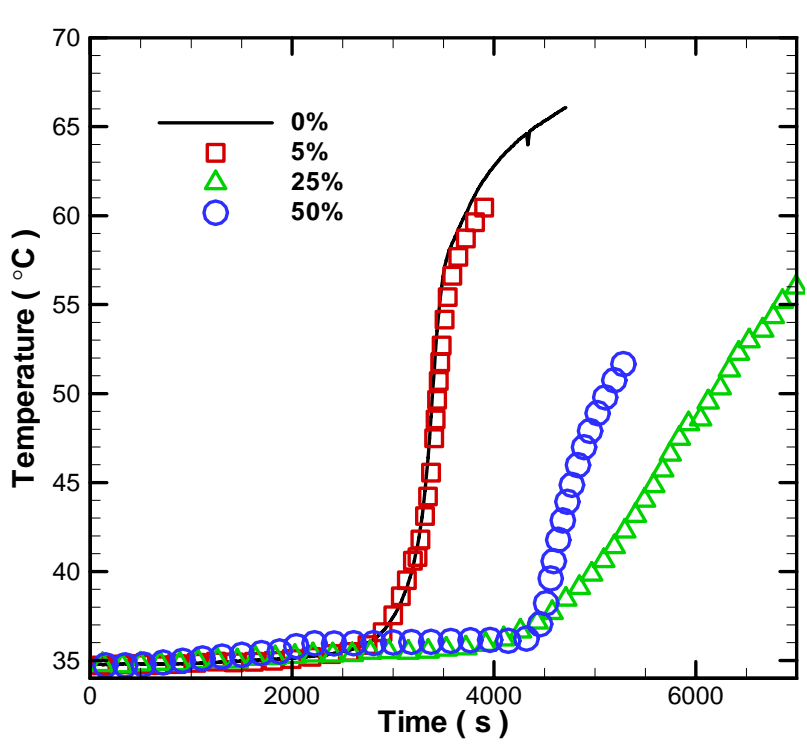

(a) B1

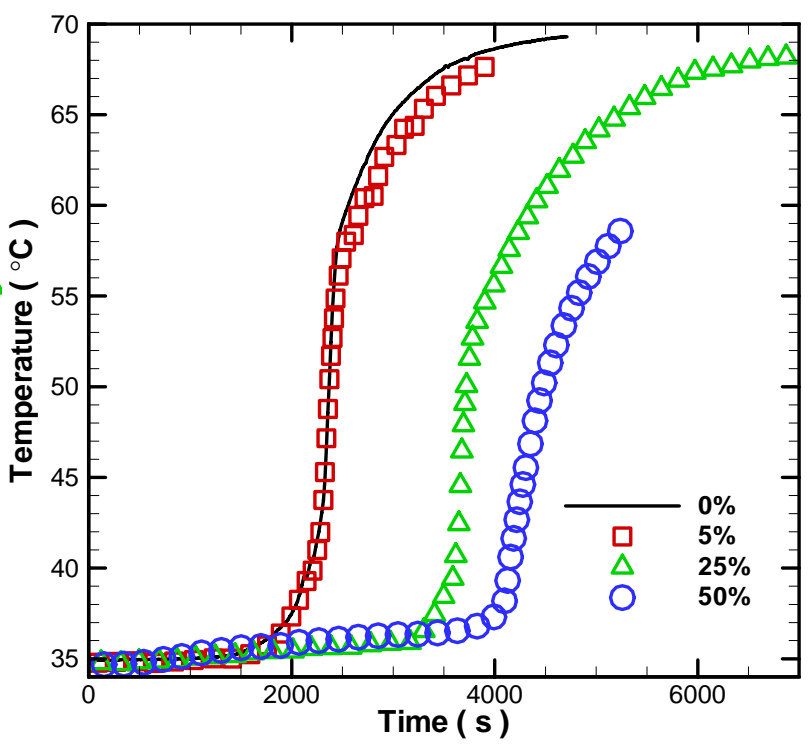

(b) D1

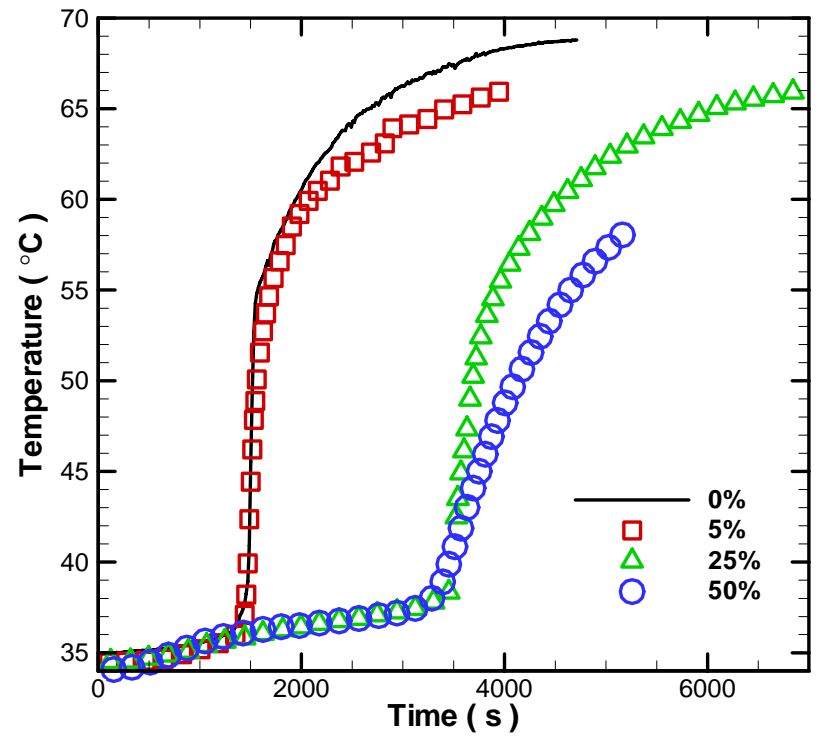

(c) F1

Figure 5. Comparison of temperature histories along the center TC rakes, B1, D1 and F1 (see Figure 1b), for cases with different particle loadings. 


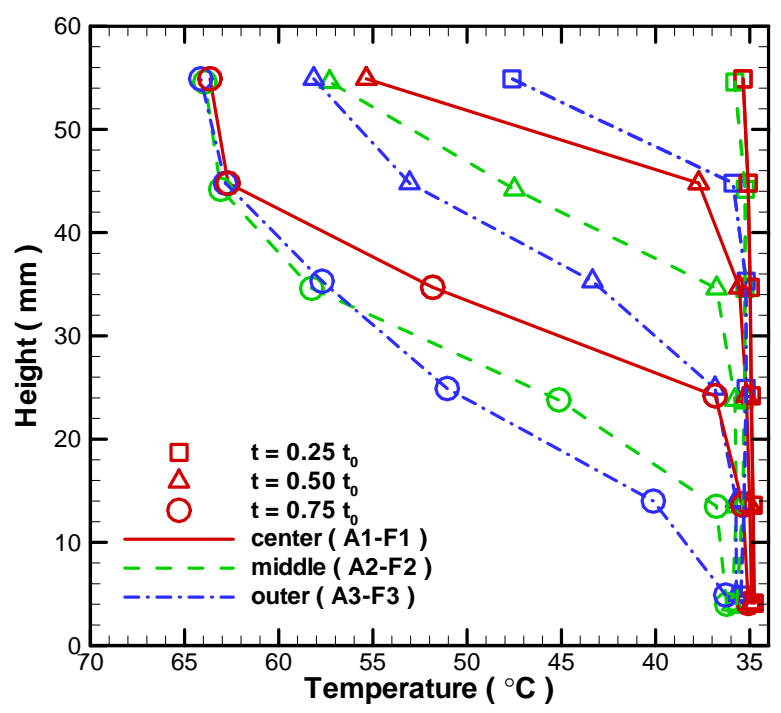

(a) $\phi=0 \%$

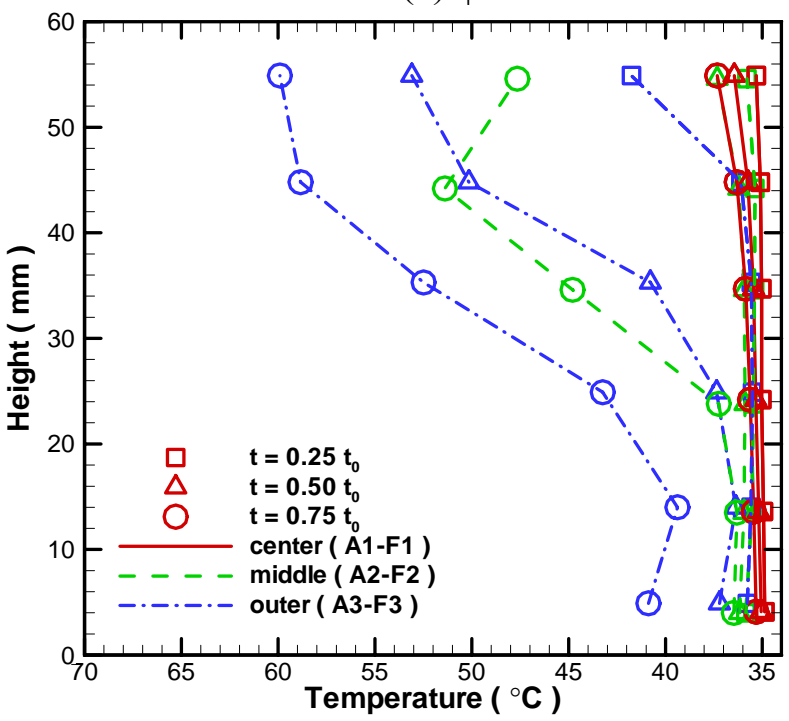

(c) $\phi=25 \%$

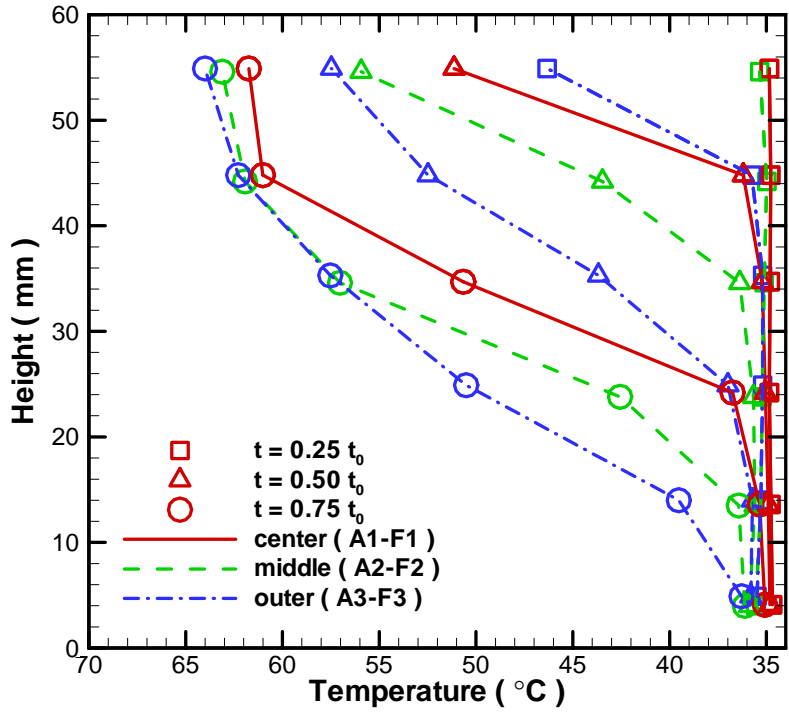

(b) $\phi=5 \%$

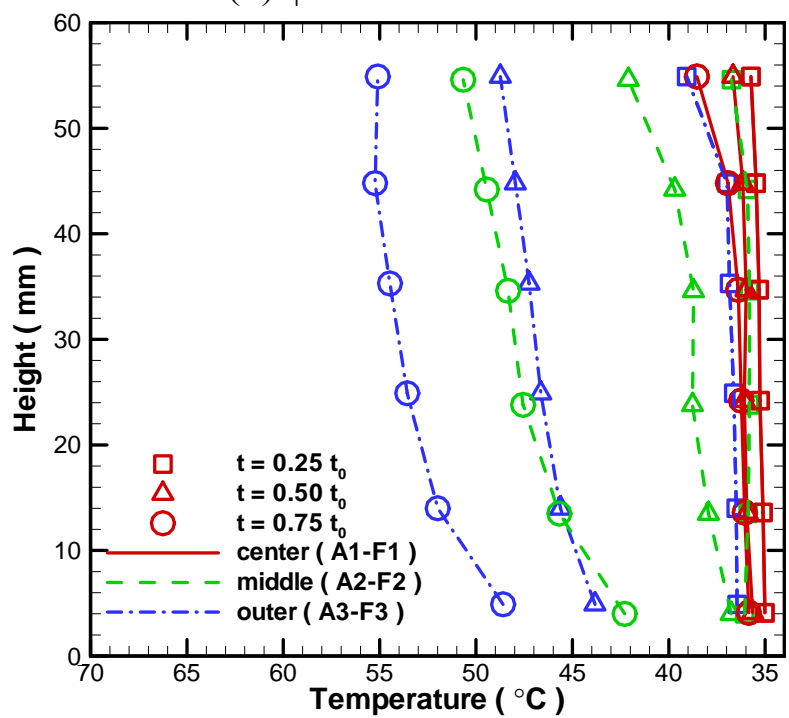

(d) $\phi=50 \%$

Figure 6. Transient temperature profiles along different thermocouple rakes for cases with different particle loadings, (a) $\phi=0 \%$, (b) $\phi=5 \%$, (c) $\phi=25 \%$, and (d) $\phi=50 \%$. 


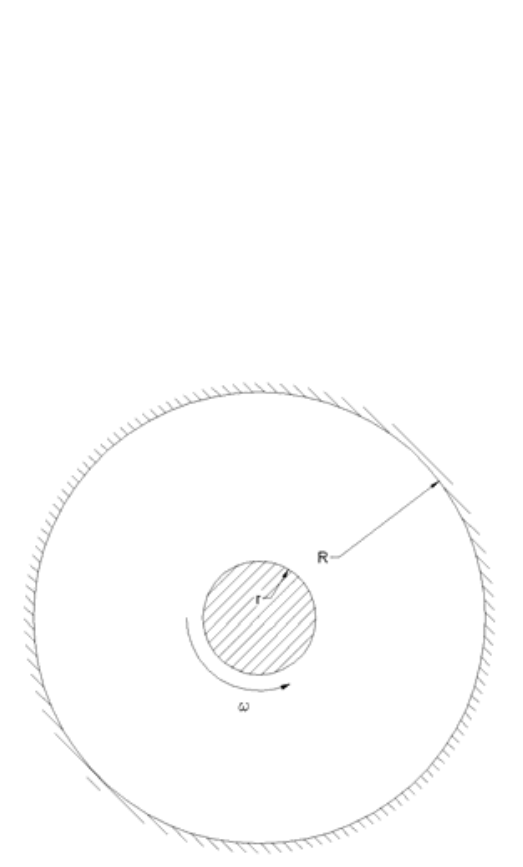

(a)

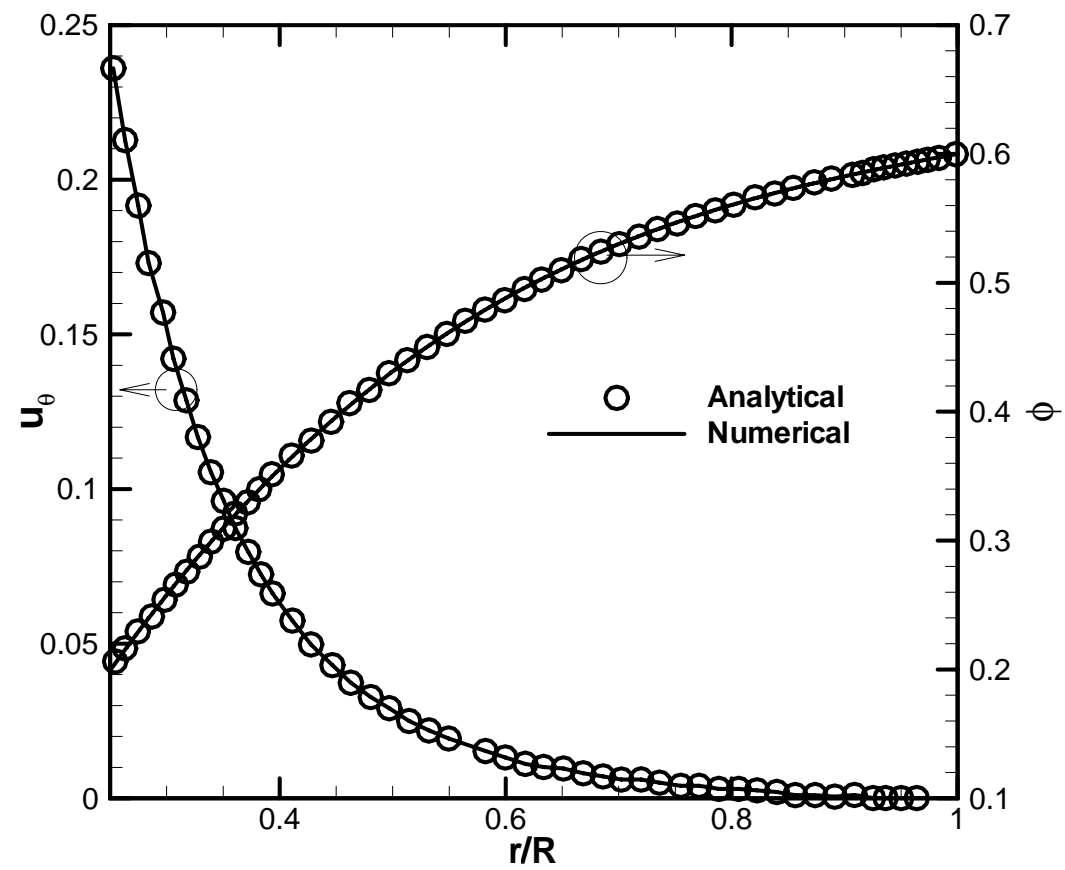

(b)

Figure 7. (a) Schematic diagram of the circular Couette flow problem, and (b) comparison between analytical and predicted velocity and particle distribution profiles. 


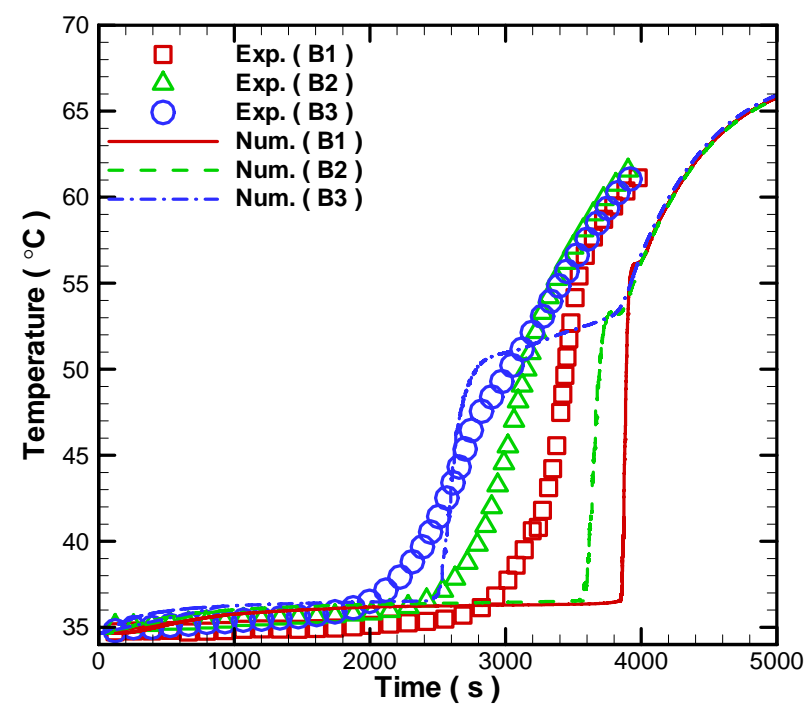

(a) B1-B3

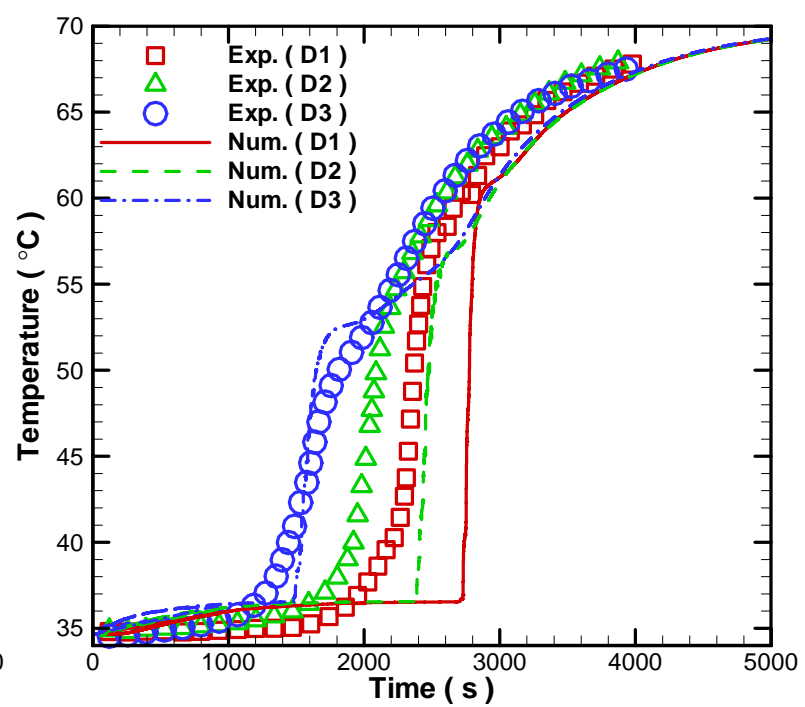

(b) D1-D3

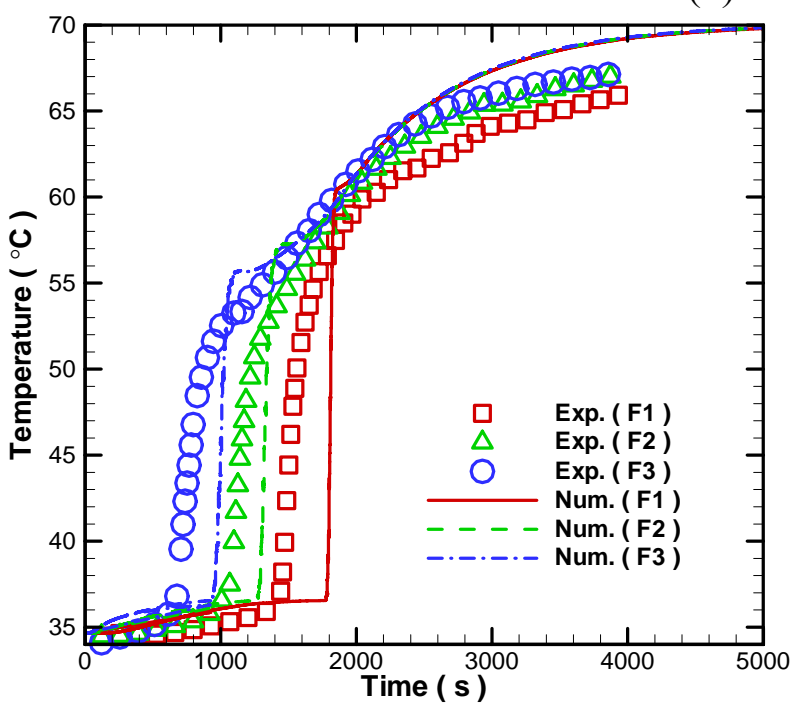

(c) F1-F3

Figure 8. Comparison of predicted and measured temperature variations at selected TC locations for $\phi=5 \%$. 


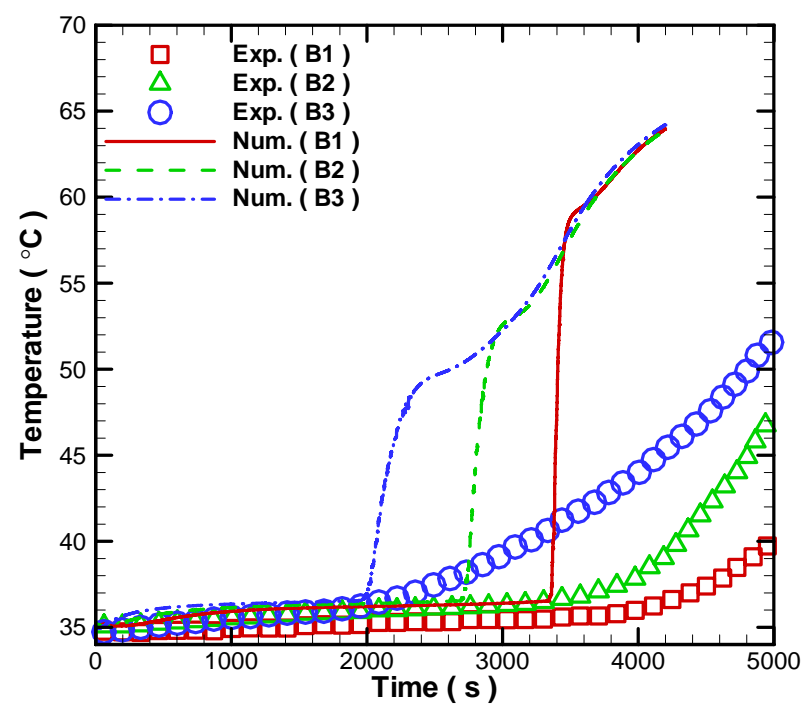

(a) B1-B3

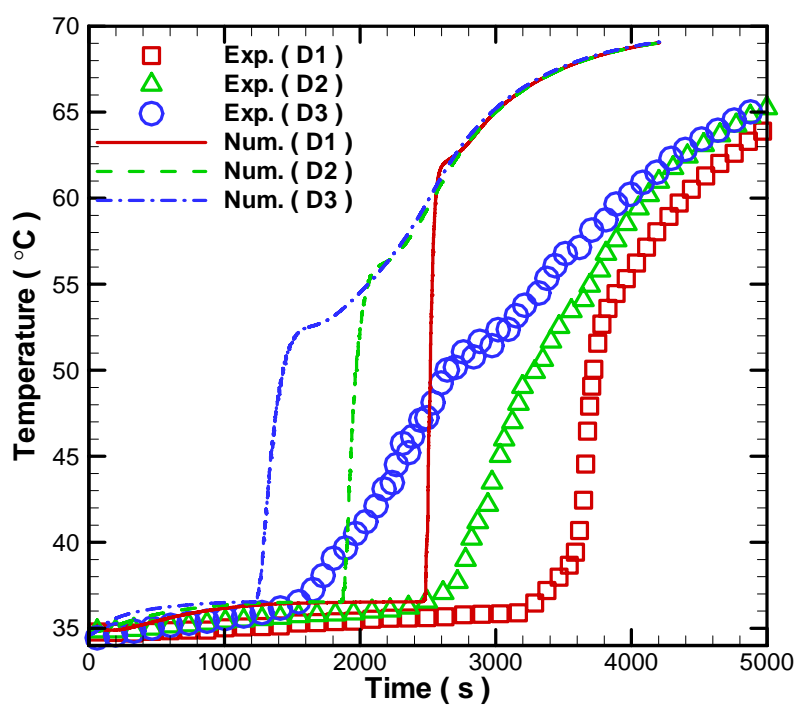

(b) D1-D3

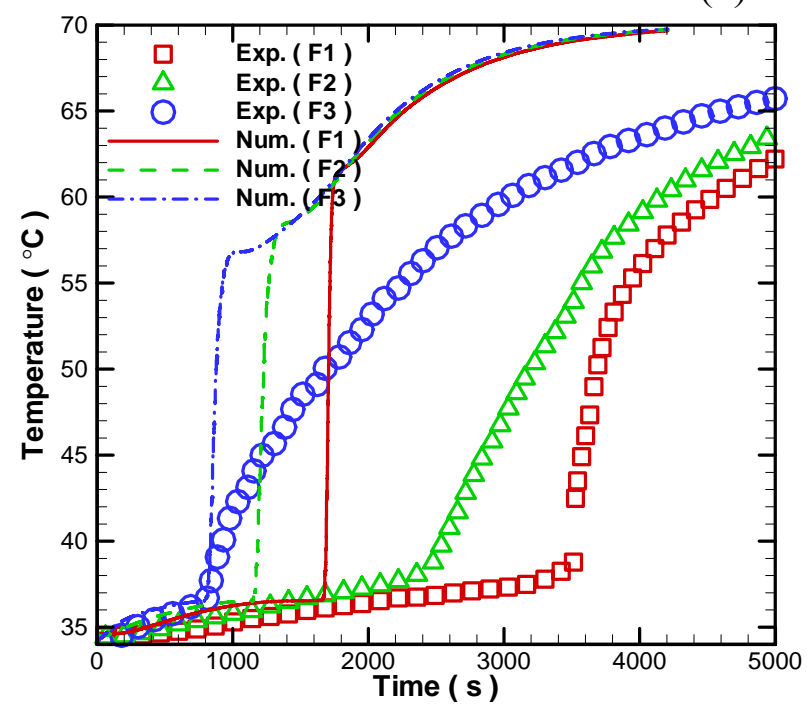

(c) F1-F3

Figure 9. Comparison of predicted and measured temperature variations at selected TC locations for $\phi=25 \%$. 


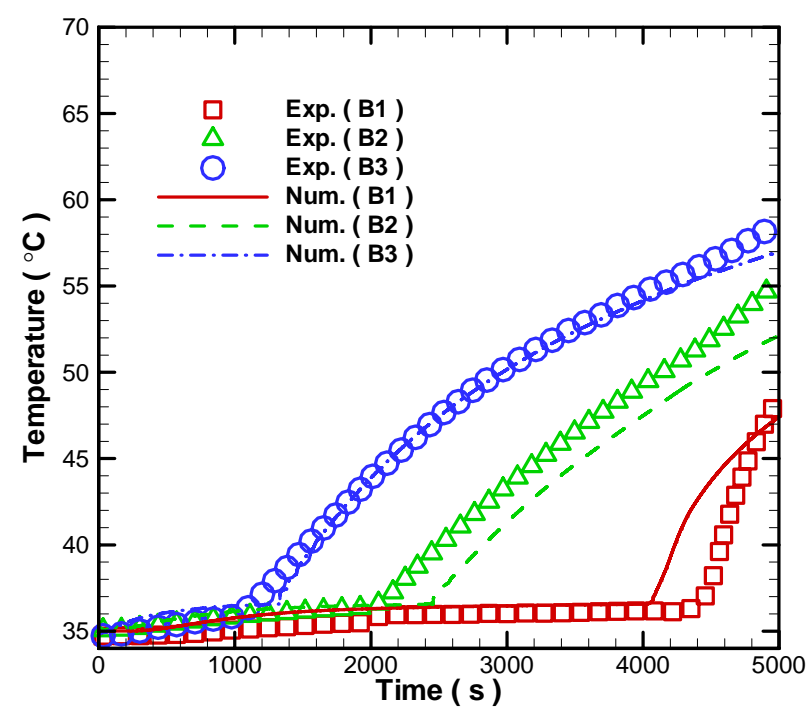

(a) B1-B3

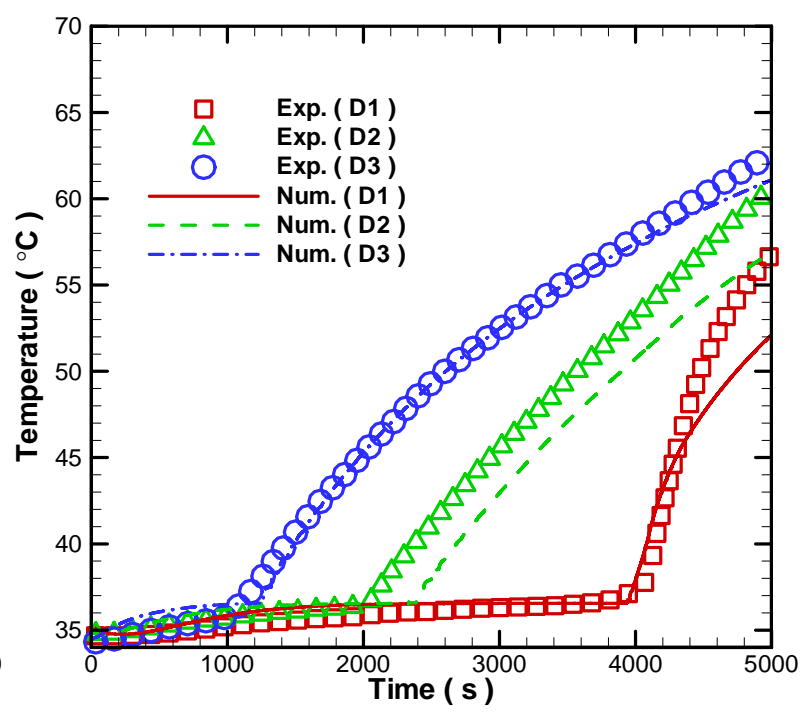

(b) D1-D3

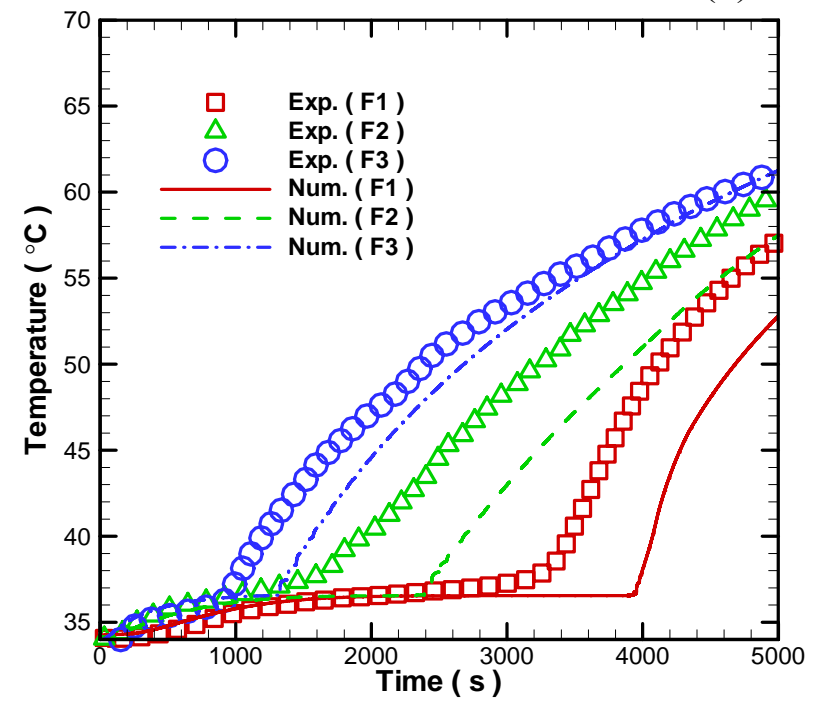

(c) F1-F3

Figure 10. Comparison of predicted and measured temperature variations at selected TC locations for $\phi=50 \%$. 